\title{
Formação de moderadores de CdP: o caso da Educação Permanente dos profssionais do Sistema Único de Saúde
}

\author{
Andrea Cristina Lovatto Ribeiro - andrea@communitas.org.br \\ Instituto Communitas \\ Nilva L. Rech Stedile - nilvarech.s@gmail.com \\ Instituto Communitas; Universidade de Caxias do Sul (UCS) \\ João L. Tavares da Silva - joaoluis@communitas.org.br \\ Instituto Communitas \\ Alexandre M. Ribeiro- aribeiro@communitas.org.br \\ Instituto Communitas
}

\begin{abstract}
Resumo. Ações em nível nacional de Educação Permanente em Saúde (EPS), baseadas no conceito de aprendizagem social por meio de Comunidades de Prática (CdPs), estão sendo as respostas inovadoras à necessidade de formação de milhares de profssionais atuantes no SUS. Este trabalho propõe um framework conceitual como subsídio para desenvolver a capacitação de e-moderadores dessas comunidades, o qual é apresentado por meio de um Ciclo de Moderação em CdPs, composto por quatro etapas. Este framework está sendo utilizado e se mostrado efcaz na formação de aproximadamente 150 moderadores que estão acompanhando comunidades de práticas de aproximadamente 2200 tutores em um curso de aperfeiçoamento semi-presencial, em um projeto de EPS para os profssionais do SUS, de abrangência nacional.
\end{abstract}

Palavras-chave: educação permanente em saúde; comunidades de prática; formação de e-moderadores

Abstract. Initiatives in Permanent Education in Health (EPS), at nacional level, based on the concept of social learning through Communities of Practice, have been the innovative responses to the need to train thousands of professionals working in the Brazilian Unifed Health System. This study proposes a conceptual framework as an aid to develop forms of training CoPs e-moderators of these communities, which is presented in the form of a Communities of Practice Moderation cycle, which comprises four steps. The Framewok is been used, and it has shown its effectiveness, in a training program for approximately 150 moderators who are following communities of practice of about 2,200 tutors on a semi-presence course, on a EPS project for SUS professionals, nationwide.

Key-works: permanent education in health; communities of practice; e-moderator's training

\section{Introdução}

O Sistema Único de Saúde (SUS), proposto na Constituição de 1988 e consolidado na Lei 8080 (Brasil, 1990) que o instituiu em 1990, tem como um dos princípios norteadores a integralidade em saúde. Um dos maiores desafos para o alcance de um cuidado integral em saúde no Brasil é a formação dos profssionais, que tem tido historicamente como base o modelo biomédico hegemônico de cuidado e a ênfase nas especialidades (Ceccim et al., 2008, Guareschi et al., 2009). Essa formação não prepara os profssionais para atenderem às demandas do SUS, que dizem respeito a uma atuação em equipes interdisciplinares, atuando na prevenção e promoção à saúde . Para dar conta das necessidades de saúde da população e do preparo profssional que o possibilita duas iniciativas são fundamentais: a necessidade do próprio SUS frmar parcerias com agentes formadores, em nível de graduação e de pós-graduação e; propor novos enfoques para a formação por meio de ações de Educação Permanente em Saúde (EPS). 
A EPS tem como ponto de partida a prática dos profssionais (Ceccim, 2005; Tesser et al., 2011) e pode ser entendida como "o processo educativo que coloca o cotidiano do trabalho - ou da formação - em saúde em análise, que se permeabiliza pelas relações concretas que operam realidades e que possibilita construir espaços coletivos para a refexão e avaliação de sentido dos atos produzidos no cotidiano". (Ceccim, 2005, p. 161). Para Tesser et al. (2011, p. 4296), a educação permanente "é fundamentada na concepção de educação como transformação e aprendizagem signifcativa, centrada no exercício cotidiano do trabalho e na valorização deste como fonte de conhecimento".

Com o advento dos recursos tecnológicos, existem ferramentas inovadoras que, além de permitir grande abrangência nas ações de EPS, possibilitam que a aprendizagem seja feita de forma colaborativa. Uma dessas possibilidades é a aprendizagem social através de Comunidades de Práticas (CdPs), nas quais o moderador ocupa papel fundamental e, para que possa exercer de forma efetiva o seu trabalho, precisa estar bem capacitado. Este trabalho propõe um framework conceitual defnindo um ciclo de moderação (CdM) para capacitação de e-moderadores no contexto de aprendizagem colaborativa usando Comunidades de Prática.

O texto está organizado da seguinte forma: inicialmente é feita a fundamentação de Comunidades de Prática e Aprendizagem Social, seguida da apresentação do papel do E-moderador na aprendizagem colaborativa, para então apresentar a proposta do framework conceitual defnindo o ciclo de moderação e um estudo de caso onde este framework é aplicado.

\section{Comunidades de prática e aprendizagem social}

A fundamentação para o uso de Comunidades de Prática como suporte à educação decorre, principalmente, dos estudos que mostram que a aprendizagem é um ato social e que no contexto atual da Sociedade do Conhecimento ela passa a ser um processo permanente, permeando o dia a dia de todas as pessoas, em especial no ambiente profssional. A Aprendizagem Social é considerada por Woolf (2010) um dos desafos da educação. Wenger (2000) diz que os “aprendizes sociais" são receptores e produtores de conhecimento, ou seja, os papéis são dinâmicos e fuidos. Hart (2009) considera que a Aprendizagem Social é a evolução natural do e-learning. Bandura (1977) descreve a Aprendizagem Social como a aprendizagem colaborativa que se dá por meio do contato e associações regulares com outros indivíduos.

O termo Comunidades de Prática foi utilizado inicialmente pelo teórico organizacional Etienne Wenger (Wenger, 2008), como sendo um grupo de pessoas que compartilha um interesse sobre um assunto ou problema e aprende com interações regulares. Mais que comunidades de aprendizes, a CdP pode ser uma "comunidade que aprende", pois é composta por pessoas que têm compromisso de agregar as melhores práticas (Wenger, 2008). O conhecimento, sendo produzido de forma colaborativa nas CdPs, responde às necessidades de EPS, uma vez que essa emerge das práticas no cotidiano dos serviços, é descentralizada, ascendente (provem de demandas dos profssionais) e interdisciplinar.

\section{E-moderadores: elementos importantes à aprendizagem colaborativa}

O aprender é um ato social. Pessoas que se empenham em atividades conjuntas se ajudam mutuamente e compartilham informações, cabendo ao moderador da comunidade fomentar as relações e as trocas entre os indivíduos. A importância do moderador em uma CdP é evidenciada por diversos autores (Downes, 2006; Dias, 2008; Hinchcliffe, 2009; Quiroz, 2010; Dale, 2011) e são várias as formas de nomear essa atividade: gerente de comunidade online (Hinchcliffe, 2009); facilitador on-line ou emoderador (Dale, 2011); mediação colaborativa (Dias, 2008) e tutor (Quiroz, 2010). As atividades desse profssional tem sido associadas às funções de regulação da 
comunidade, de seus conteúdos, tendo o moderador como protagonista e não os alunos. No entanto, essa liderança deve ser partilhada entre os membros, por meio de uma mediação colaborativa. (Dias, 2008). O papel do moderador nesta abordagem é muito mais intermediar as discussões e facilitar a interação, do que dar respostas diretas as demandas ou conferir e avaliar respostas, posicionamentos ou proposições.

Alguns autores já se debruçaram sobre o estudo das funções do e-moderador, mas não com a estruturação de um roteiro para a formação desses profssionais. Dale (2011) afrma que não se pode falar em uma comunidade com sucesso, mas uma comunidade com saúde. Sinais de que a comunidade está com saúde incluem número de membros, índice de crescimento da mesma, número e frequência de documentos carregados e "baixados", número e frequência de posts nos fóruns, número e frequência de comentários, tempo passado na CdP pelos membros, etc. Para esse autor há evidências claras de que uma CdP que conta com um ou mais moderadores é muito mais propensa a ter sucesso do que uma CdP que depende somente da auto-organização. Para Quiroz (2010), o tutor deve ter como objetivo guiar o aluno em direção a uma aprendizagem colaborativa. Dentre as suas atividades, estão: "manter vivos os espaços comunicativos, facilitar o acesso aos conteúdos, animar o diálogo entre os participantes, ajuda-los a compartilhar o seu conhecimento e a construir novos conhecimentos". (p. 14)

Dias (2008) discute a necessidade de refexão quanto ao papel do moderador, já que há em curso uma mudança de foco nos ambientes de aprendizagem virtuais: da transmissão de informações para a interação, a colaboração, o conhecimento coletivo: "a aprendizagem é sustentada pela dinâmica da rede nos contextos de construção colaborativa dos objetos e narrativas de conhecimento da comunidade, e na integração das representações sociais na elaboração de uma prática comum e partilhada" (p. 4). O papel do moderador, nesse sentido, deve passar de regulador e "líder" da comunidade, no primeiro modelo de ambientes virtuais de aprendizagem, para uma mediação colaborativa, no segundo modelo, onde a liderança é devolvida a comunidade.

Hinchcliffe (2009) propõe áreas de atuação do Gerente de Comunidade: gerenciamento da plataforma, de projetos, de produtos, de clientes e da marca, o desenvolvimento profssional e da equipe e o gerenciamento de conteúdos e da comunidade. A moderação propriamente dita faz parte do gerenciamento da comunidade. Nesse modelo, as atividades e responsabilidades de um Gerente de Comunidade vão muito além das atividades esperadas de um e-moderador em comunidades de práticas voltadas para a EPS.

Dias (2008) traz contribuições importantes, afrmando que os ambientes virtuais de aprendizagem estão mudando de uma abordagem instrucional tradicional para a aprendizagem colaborativa e que o papel do moderador deve acompanhar essa mudança. É o que é denominado por este autor de mediação colaborativa, uma forma de atuação desse profssional coerente com as características da aprendizagem colaborativa. As refexões propostas por Dias (2008) são relevantes na medida em que apontam novas possibilidades para a atuação desse profssional e enfatizam o papel central do mesmo. Porém, para a organização de um processo de formação para emoderadores, no caso de moderadores para atuação em ações de EPS, foi necessário construir um novo modelo de estrutura, que compreendesse todo o processo de moderação, desde a preparação desses profssionais para o exercício das atividades de moderação, para a moderação da comunidade propriamente dita e para a avaliação das ações e do andamento da comunidade. É relevante salientar que os moderadores a serem capacitados eram um grupo heterogêneo: muitos deles eram profssionais da área da saúde com diferentes cursos e níveis de familiaridade com tecnologias para a educação; alguns com experiência em ambiente virtuais de aprendizagem, outros não. 


\section{Proposta de um framework conceitual para e-moderadores em CdP: o Ciclo de Moderação em CdP}

Conforme demonstrado acima o papel do e-moderador é fundamental para a aprendizagem colaborativa em comunidades. Os estudos sobre o papel desses profssionais são recentes e há a necessidade de capacitação dos mesmos. A partir da defnição das competências desse profssional, das características de um modelo de aprendizagem social usando CdPs e da necessidade de uma boa mediação colaborativa, este trabalho propõe um framework conceitual para desenvolver as formas de capacitação do e-moderador.

Em essência, um framework conceitual, apresenta um corpo de conceitos interrelacionados que defne uma interpretação ideológica de uma solução concreta (Miles e Huberman, 1994; Botha, 1989; Shields, 2013; Najam, 1996). Pode ser utilizada como uma ferramenta analítica que fundamenta um conjunto de regras, formalismos, conceitos e métricas que defnem um método ou metodologia de aplicação. Pode ser convertido em modelos abstratos de software (Johnson 1997) ou simplesmente organizar um corpo de pesquisa para a construção de um produto, artefato ou metodologia (Levering, 2002). Neste trabalho, o corpo de conceitos e fatores de desenvolvimento das competências específcas para um e-moderador foi desenvolvido e apresentado na forma de um Ciclo de Moderação em CdPs, composto por 4 etapas, com a fnalidade de subsidiar a capacitação desses atores.

Conforme ilustrado na Figura 1, o trabalho do e-moderador começa em uma etapa anterior ao início do trabalho de e-moderação propriamente dito, passando pelas etapas de moderação da aprendizagem social, avaliação da evolução da CdP e redefnição de estratégias após essa avaliação.

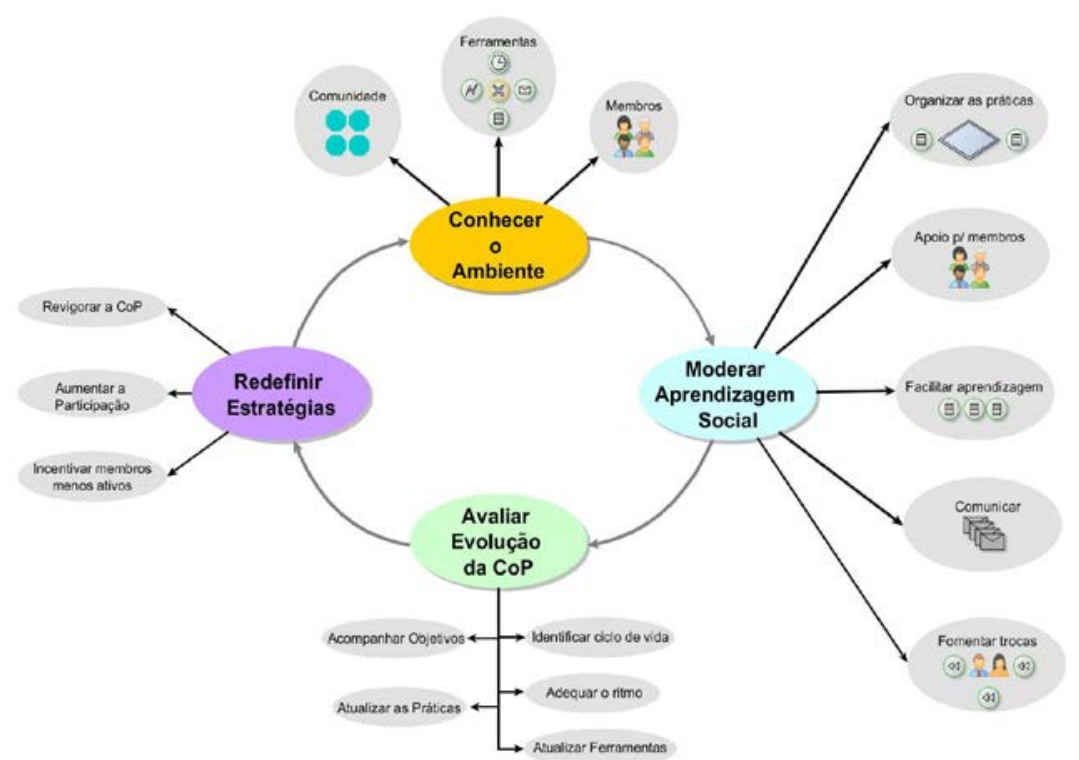

Figura 1- Ciclo de vida do e-moderador em Comunidades de prática para Educação Permanente

Fonte: os autores

As etapas constitutivas do processo de moderação das CdP, conforme Figura 1, são: conhecer o ambiente; moderar processos de aprendizagem social; avaliar o andamento/ processo de evolução da Comunidade e redefnir estratégias de acordo com os resultados da avaliação. 


\subsection{Conhecer o ambiente}

Conhecer o ambiente é a etapa inicial do ciclo de moderação e é anterior ao início da moderação de CdPs. É uma etapa de preparação por parte do futuro moderador. Embora esta etapa seja inicial ela não se completa antes do início da moderação, ou seja, a apropriação do ambiente se dará de forma contínua, ao longo de todo o processo. Nessa etapa, ele deve conhecer quem são os membros da comunidade; conhecer as características principais da comunidade da qual ele será o moderador e conhecer as ferramentas disponíveis para uso na comunidade.

\section{a) Conhecer os membros}

Conhecer o perfl dos membros da comunidade é uma parte importante da preparação do moderador para a sua atuação nessa função. O moderador deve procurar identifcar quais são os interesses dos membros da comunidade, bem como as suas necessidades. Sabendo o que os membros estão buscando na comunidade, o facilitador responderá de forma mais efetiva às necessidades dos mesmos. Dentre as características que constituem o perfl dos membros estão por exemplo a idade, formação e a atuação profssional. A partir da identifcação dessas características, o moderador deve adequar a sua linguagem ao público da comunidade.

\section{b) Conhecer a Comunidade de Prática}

Para preparar-se para o processo de moderação da aprendizagem social em CdPs, além de conhecer o perfl dos membros, o moderador deve conhecer as características mais importantes da comunidade. Dentre essas características estão: identifcar o domínio da comunidade, identifcar as práticas da comunidade e identifcar o estilo/orientação da mesma (Wenger et al., 2009). Conhecendo essas características, o moderador estará mais preparado para auxiliar os membros a manter o foco nos objetivos em torno dos quais a comunidade foi criada e a propor estratégias para auxiliálos a alcançar esses objetivos.

\section{c) Conhecer as ferramentas}

Conhecer as ferramentas disponíveis para uso em CdP é a terceira parte da etapa preparatória para a moderação de comunidades. Nesse momento, o moderador deve ser capacitado para conhecer as potencialidades dos recursos tecnológicos para a educação; identifcar tecnologias que facilitam o processo de aprendizagem; conhecer as ferramentas utilizadas em CdPs e identifcar as ferramentas que são necessárias e adequadas para cada CdP, de acordo com as suas características singulares. $\mathrm{O}$ moderador deve ser incentivado a atualizar-se em relação as tecnologias e a buscar auxílio de especialistas quando necessário.

Essa etapa que antecede ao trabalho do moderador propriamente dito, que aqui chamamos de "Conhecer o ambiente", não foi descrita nas outras sistematizações do papel do moderador citadas anteriormente. Trata-se de uma etapa importante que prepara e dá subsídios para a atuação do profssional. Os futuros moderadores precisam preparar-se para conhecer os membros, a Comunidade e as ferramentas da(s) comunidade (s) que irá moderar.

\subsection{Moderar aprendizagem social}

Conhecendo o perfl dos membros da comunidade, as características principais da comunidade e as ferramentas disponíveis, o moderador estará melhor preparado para moderar a aprendizagem social em uma CdP.

Para moderar de forma efciente a aprendizagem social em CdPs, o moderador deve ser capacitado para: a) ser um ponto de apoio para os membros; b) fomentar as trocas entre os membros da Comunidade; c) auxiliar no processo de aprendizagem dos 
membros; d) organizar as práticas e e) comunicar-se clara e efcientemente em Comunidades de Prática.

\section{a) Ser um ponto de apoio para os membros}

O moderador deve ser considerado um ponto de apoio para os membros da comunidade. Algumas ações por parte do moderador fazem com que isso aconteça, dentre elas: mostrar-se disponível/acessível para atender as necessidades dos membros; estabelecer um vínculo com membros da comunidade e tratar as questões dos usuários com atenção, dando respostas adequadas em termos de conteúdo e tempo.

\section{b) Fomentar as trocas entre os membros da Comunidade}

Uma das tarefas mais importantes do moderador é auxiliar os membros a desenvolverem a capacidade de aprender de forma colaborativa. Para isso, o moderador da comunidade deve incentivar a troca de experiência e conhecimento entre os membros, incentivar o trabalho em equipe e a construção conjunta de conhecimento utilizando as ferramentas disponíveis para uso na comunidade, incentivar a interação regular, encorajar o compartilhamento de ideias. Para que isso seja possível, é importante que o moderador ajude a criar um clima de confança entre os membros da comunidade. Cabe destacar que se a comunidade está interagindo intensamente ou algum membro da comunidade assume este papel momentaneamente, o moderador pode acompanhar o processo, sem necessariamente intervir diretamente no seu andamento.

\section{c) Auxiliar no processo de aprendizagem dos membros}

O moderador de aprendizagem social em CdPs deve ser capaz de auxiliar os membros no seu processo de aprendizagem. Dentre as ações do moderador estão: identifcar atividades de aprendizagem importantes para a comunidade; propor atividades de aprendizagem que atendam as necessidades dos membros; instigar a pesquisa; auxiliar a comunidade a criar um clima propício para a aprendizagem, com base no respeito mútuo e na confança e auxiliar no estabelecimento de prioridades nas atividades a serem desenvolvidas, mediar confitos entre os membros.

\section{d) Organizar as práticas}

Umas das funções do moderador é organizar as práticas da comunidade. Dentre as atividades feitas pelo moderador com esse objetivo podem ser elencadas: ordenar, estruturar e sistematizar materiais disponibilizados; organizar documentos que a comunidade compartilha; organizar, estruturar e sistematizar trabalhos em grupo no ambiente virtual; auxiliar na defnição da forma de organização das atividades; auxiliar na defnição das formas de acesso ao conhecimento e organização das fontes de conhecimento; e trabalhar no sentido de fazer com que a comunidade torne-se uma fonte de conhecimento para seus membros.

\section{e) Comunicar-se em Comunidades de Prática}

Para que o moderador possa realizar o seu trabalho é importante que ele consiga comunicar-se adequadamente. Em CdPs, isso signifca que ele deve ser capaz de comunicar-se de forma síncrona e assíncrona com membros da comunidade; comunicar-se com clareza e objetividade; adequar a linguagem utilizada de acordo com perfl dos membros; saber ouvir. Pela importância da capacidade de se comunicar para um moderador, esse deve ser um dos elementos presentes no momento da capacitação desses profssionais. 


\subsection{Avaliar o andamento/ processo de evolução da Comunidade}

Após o início do processo de moderação das CdPs, uma das funções do moderador é a de avaliar o andamento e o processo de evolução da comunidade. Para isso é importante que o moderador seja capaz de: identifcar a etapa do ciclo de vida em que a comunidade se encontra e atuar de acordo; analisar o andamento da Comunidade e agir para um melhor aproveitamento da mesma pelos membros; identifcar a adequação das ferramentas utilizadas pela Comunidade; identifcar se os objetivos da Comunidade estão sendo alcançados; identifcar oportunidades para sugestão de novas atividades de aprendizagem de acordo com necessidades e interesses dos membros, e ainda, com base em Dale (2011), avaliar a "saúde" da comunidade.

\subsection{Redefnir estratégias de acordo com os resultados da avaliação}

Após avaliar o andamento/ processo de evolução da comunidade, o moderador deve utilizar estratégias para "recuperar a saúde" da comunidade e motivar a participação, quando identifcada essa necessidade. Alguns autores sugerem estratégias possíveis para serem utilizadas por moderadores como, por exemplo, Dale (2011) e Parboosingh (2010).

Alguns exemplos de estratégias sugeridas por Dale (2011) serão descritas a seguir. Quando for identifcado que não há participação ou atividade na comunidade, o moderador pode postar novos conteúdos, solicitando feedback e comentários, com o objetivo de estimular novas conversas. No caso da participação na comunidade por apenas algumas pessoas, uma ação por parte do moderador pode ser a de telefonar ou enviar e-mail para os membros que não participaram por algum tempo, procurando descobrir o motivo. Nessa situação Dale (2011) sugere que o moderador deve certifcarse que os membros menos ativos sabem como utilizar as ferramentas.

\section{Um caso de Formação de Moderadores usando o ciclo de moderação}

O CdM proposto acima está sendo utilizado como base para a formação de 150 e-moderadores, provenientes das 5 regiões brasileiras, no Programa "Caminhos do Cuidado", promovido pelo Ministério da Saúde. Esse programa tem como foco a formação de profssionais da saúde para atuar com a dependência à drogas, mais especifcamente para lidar com a política de redução de danos. O Caminhos do Cuidado é um programa do Governo Federal que visa à formação de 300.000 Agentes Comunitários de Saúde (ACSs) e Técnicos de Enfermagem na temática de álcool e drogas, em especial do crack, em todo o território Nacional. Dentro deste programa, a formação dos Orientadores (150) e dos Tutores (2200) é realizada em um modelo blended, sendo que os Orientadores recebem uma formação específca para atuarem como e-moderadores nas CdPs com os respectivos grupos de Tutores. Esses, por sua vez, são responsáveis pela capacitação dos ACSs e técnicos de Enfermagem

Para contemplar as necessidades de formação dos moderadores, em todas as etapas constitutivas do CdM, foi formada uma equipe interdisciplinar de formadores. A equipe foi composta por profssionais da área da psicologia, da educação, da enfermagem e da informática na educação. O conteúdo do curso foi elaborado de forma a contemplar todas as atividades descritas no ciclo.

A formação foi realizada em grupos, variando de 30 a 40 pessoas, com duração de 8 horas presenciais e horas adicionais pela comunidade conforme necessidades específcas. Assim, cada grupo teve a sua formação realizada em três etapas: uma etapa presencial conceitual, focada nos aspectos metodológicos; uma etapa presencial referente ao uso das tecnologias e ferramentas; e uma etapa usando uma CdP em um modelo de Educação Permanente, onde as situações vivenciadas no cotidiano do trabalho são discutidas pelo grupo, revisando as práticas correntes, compartilhando experiências e construindo novas práticas quando necessário. 
O enfoque da formação, usando o ciclo de moderação apresentado, capacita os moderadores a refetirem sobre ferramentas tecnológicas e suas respectivas formas de uso e a se familiarizarem com a Comunidade de Práticas (Etapa 1 - Conhecer o Ambiente), já que as várias orientações e objetivos de uma comunidade podem requerer diferentes confgurações e usos de ferramentas tecnológicas. Neste aspecto, a formação contou com um módulo prático de ofcina tecnológica na plataforma de CdP utilizada pelo Programa.

Aspectos metodológicos e pedagógicos de aprendizagem sócio-interacionista são abordados sob a ótica do compartilhamento de conhecimento usando o ferramental tecnológico defnido. Portanto, a Etapa 2 (Moderar a Aprendizagem Social) foca na capacidade do moderador de resolver confitos de interação e de conteúdo e de incentivar a aprendizagem colaborativa e o protagonismo dos membros da comunidade no próprio processo de aprendizagem. Com isto, os e-moderadores conseguiram mapear as atividades pedagógicas com as ferramentas adequadas a fm de maximizar o resultado desejado. Nessa etapa foram utilizados estudos de caso, nos quais foram apresentadas intervenções não adequadas realizadas por $e$-moderadores em CdPs e os $e$-moderadores em processo de capacitação foram convidados a analisar as situações apresentadas e propor outras formas de intervenção.

A partir do acompanhamento sistemático das interações na CdP, o moderador pode "Avaliar a Evolução da CdP" (Etapa 3) e medir se os resultados produzidos estão sendo realimentados na comunidade. Funcionalidades tecnológicas de monitoramento da participação ajudam os moderadores a gerenciar as atividades da CdP e acompanhar as discussões e comentários em Fóruns, diálogos e criação de conteúdo. Desta forma, uma análise da "saúde vital" da comunidade é apresentada aos moderadores para que eles possam diagnosticar problemas e intervir usando novas estratégicas de participação (Etapa 4 - Redefnir Estratégias).

A construção do CdM e a estruturação do programa de formação com base nele possibilitou que a capacitação desses profssionais abordasse todos os aspectos relacionados a moderação de CdPs utilizadas na EPS, desde a preparação para esse trabalho até a avaliação do mesmo. Além disso, todo o avanço conceitual, os conhecimentos sistematizados e disponibilizados ao grupo fcam devidamente registrados, possibilitando um revisitar constante da comunidade de prática e de seus avanços conceituais.

\section{Considerações Finais}

Conforme apresentado na introdução, a Reforma Sanitária Brasileira trouxe a tona um problema: os profssionais da saúde haviam sido formados até então em uma lógica centrada no atendimento médico e individual, o que não correspondia as necessidades do "novo" Sistema Único de Saúde. Assim, para dar conta da formação dos profssionais que já estavam em serviço foi instituída a Política Nacional de Educação Permanente em Saúde (Brasil, 2006), a partir da qual várias iniciativas de formação profssional tem sido desenvolvidas. Cabe destacar que o Brasil tem dimensão continental o que justifca o uso de TICs para este propósito.

O CdM proposto pode ser considerado um Framework conceitual utilizado como base para o desenvolvimento da Capacitação de e-moderadores. Foi elaborado a partir do exame de cada um dos elementos propostos no "ciclo" e da comparação desses elementos conceituais com modelos disponíveis.

Foram discutidos neste artigo aspectos referentes ao papel dos e-moderadores numa perspectiva de Aprendizagem Social em Comunidades de Prática, o qual é diferente do papel tradicional na medida em que a este cabe mais colaborar e mediar discussões e análises, do que dar respostas prontas e avaliar a aprendizagem por meio de provas. O CdM como guia para a realização de ofcinas de formação de emoderadores num modelo blended, mostra-se uma ferramenta importante para o 
entendimento desse papel e, consequentemente, para o exercício das novas competências necessárias nos processos de aprendizagem colaborativa.

O ciclo de moderação proposto e utilizado nas ofcinas de formação de emoderadores do Caminhos do Cuidado se mostrou efetivo, servindo como referência para o desenvolvimento da formação e tendo dado uma boa base inicial para esses profssionais atuarem nas respectivas comunidades, segundo os depoimentos dos próprios participantes.

Como trabalhos futuros, pretende-se avaliar de forma mais sistemática como os e-moderadores colocam em prática o CdM utilizado na sua própria formação, por meio de algum tipo de indicador de participação e conteúdo gerado na comunidade.

\section{Referências}

BANDURA, A. Social Learning Theory. New York: General Learning Press, 1977. BOTHA, M.E. Theory Development in Perspective: The Role of Conceptual Frameworks and Models in Theory Development. Journal of Advanced Nursing v.14, n.1, p. 49-55, 1989.

BRASIL. Lei $\mathbf{n}^{\mathbf{0}} \mathbf{8 0 8 0} / \mathbf{1 9 9 0}$. Dispõe sobre as condições para a promoção, proteção e recuperação da saúde, a organização e o funcionamento dos serviços correspondentes e dá outras providências. Presidência da República, 1990.

BRASIL. Ministério da Saúde. Política Nacional de Educação Permanente em Saúde. $\quad$ B r a síl i a , $\quad 2006$ D i s p o níve l $\quad$ e m http://www.aids.gov.br/sites/default/fles/16_politica_nacional_eps.pdf. Acesso em 01 out. 2014.

CECCIM, R.B. Educação Permanente em Saúde: desafo ambicioso e necessário. Interface, Botucatu, n.16, p. 161-168, 2005. Disponível em <http://www.scielo.br/scielo.php?script=sci_arttext\&pid=S1414$32832005000100013 \& \operatorname{lng}=$ en $\&$ nrm=iso $>$. Acesso em 25 abr. 2012.//dx.doi.org/10.1590/S1414-32832005000100013.

CECCIM, R.B. et al . Imaginários da formação em saúde no Brasil e os horizontes da regulação em saúde suplementar. Ciência e saúde coletiva, Rio de Janeiro, v. 13, n. 5, p. 1567-1578, oct. 2008. Disponível em <http://www.scielo.br/scielo.php? script=sci_arttext\&pid=S1413-81232008000500021\&lng=en\&nrm=iso $>$. Acesso em 10 out. 2011.

DALE, S. The Role of the Facilitator in Building and Sustaining Communities of Practice. 2011. Disponível em: http://steve-dale.net/2011/09/19/the-role-of-thefacilitator-in-building-and-sustaining-communities-of-practice/ Acesso em 10 jul. 2014.

DIAS, P. Da e-moderação à mediação colaborativa nas comunidades de aprendizagem. Educação, Formação e Tecnologias, v. 1, n.1, p. 4-10, mai. 2008.

DOWNES, S. Learning Networks and Connective Knowledge. 2006. Disponível em http://it.coe.uga.edu/itforum/paper92/DownesPaper92.pdf . Acesso em 06 jul. 2014.

GUARESCHI, N. M. F. et al . A formação em Psicologia e o profssional da Saúde para o SUS (Sistema Único de Saúde). Arquivos Brasileiros de Psicologia, Rio de Janeiro, v. 61, n. 3, p. 35-45, dez. 2009 . Disponível em <http://pepsic.bvsalud.org/scielo.php? script=sci_arttext\&pid=S1809-52672009000300005\&lng=pt\&nrm=iso $>$. Acesso em 25 out. 2011.

HART, J. From e-learning to social learning - Learning \& Skills Group Conference. 2009. Disponível em http://www.c4lpt.co.uk/articles/el2sl.html. Aceso em 11 mai. 2014. 
HINCHCLIFFE, D. Community management: The 'essential' capability of successful $\begin{array}{lllll}\text { En terprise } & 2.0 & \text { effort } & \text { Disponível e m }\end{array}$ http://www.zdnet.com/blog/hinchcliffe/community-management-the-essentialcapability-of-successful-enterprise-2-0-efforts/913. Acesso em 10 jul. 2014.

JOHNSON, R.E. Components, frameworks, patterns. Proceedings of the 1997 symposium on Software reusability (SSR '97), New York: Medhi Harandi. 1997. DOI=10.1145/258366.258378. http://doi.acm.org/10.1145/258366.258378.

LEVERING, B. Concept analysis as empirical method. International Journal of Qualitative Methods, v.1, n.1, p. 35-48, 2002.

MILES, M. B., HUBERMAN, A. M. Qualitative data analysis: An expanded source book (2 ed.). Newbury Park, CA: Sage. 1994.

NAJAM, A. GO Accountability: A Conceptual Framework. Development Policy Review, n. 14, p. 339-354, 1996. doi: 10.1111/j.1467-7679.1996.tb00112.x.

PARBOOSINGH, J. Tools for CoP Facilitators - material for CoP Workshop facilitators. 2010. Disponível em https://www.usq.edu.au/ /media/USQ/Communities \%20of\%20Practice/Parboosingh\%20tools_for_cop_facilitators-_may_1-09.ashx Acesso em 20 set. 2014.

QUIROZ, J. El rol del tutor en los entornos virtuales de aprendizaje. Innovación Educativa, v. 10, n.52, p. 13-23, jul-set, 2010. Disponível em http://www.redalyc.org/articulo.oa?id=179420763002. Acesso em 10 jul. 2014.

SHIELD, P., RANGARJAN, N. A Playbook for Research Methods: Integrating Conceptual Frameworks and Project Management. Stillwater, OK: New Forums Press. 2013.

TESSER, C. D. et al. Estratégia saúde da família e análise da realidade social: subsídios para políticas de promoção da saúde e educação permanente. Ciência e saúde coletiva. v.16, n.11, p. 4295-4306, 2011.

WENGER, E. Communities of Practice and Social Learning Systems. Organization. v. 7, n.2, p. 225-246, mai., 2000. doi: 10.1177/135050840072002 Disponível em http://org.sagepub.com/content/7/2/225.short?rss=1\&ssource=mfr. Acesso em 10 abri 2014.

WENGER, E; WHITE, N; SMITH, J.D. Digital Habitats: Stewarding Technology for Communities. CPsquare, 2009, 227 p.

WENGER, E. Communities of practice: a brief introduction. 2010. Disponível em: www.ewenger.com/theory/index.htm. Acesso: 10 jun. 2010.

WOOLF, B.P. (org). A Roadmap for Education Technology. Global Resources for Online Education (GROE). 2010. 